\title{
Diagnosis of Medically Important Viruses: A Molecular Approach
}

\author{
Konika Razdan ${ }^{1}$, Varshiesh Raina ${ }^{2}$ and Bijendra K Bajaj ${ }^{*}$ \\ 1PhD, School of Biotechnology, University of Jammu, Jammu, India. \\ 2Ph.D, Project Scientist, National Institute of Immunology, New Delhi, India. \\ ${ }^{3^{*} \mathrm{PhD}}$, Senior Assistant Professor, School of Biotechnology, University of Jammu, Jammu, India. \\ [Author $1 \& 2$ have contributed equally]
}

\begin{abstract}
Serological and molecular markers are key elements in diagnosis, prognosis and treatment of various viral diseases. Automated Molecular biology techniques have revolutionized present day viral testing and by introducing fully automated devices with faster turnaround time's clinical laboratories are able to report sensitive and accurate results to physicians leading to better patient care. Qualitative and quantitative molecular virus assays, based on PCR technology could identify active infection by detecting viral messenger RNA (mRNA) but the most widely used diagnostic tests are quantitative real-time PCR techniques. These techniques are rapid, highly sensitive, reproducible and have a broad dynamic range. This review focuses on the main nucleic acid techniques, their clinical applications, special applications and opportunities that are provided by these methods in clinical
\end{abstract}

\section{INTRODUCTION}

During the last years viral testing by Molecular diagnostic techniques has experienced a rapid development ${ }^{1}$, and a majority of laboratories have introduced it as a new way of diagnosis. Amalgamation of Molecular microbiology and automated methodology has allowed clinical laboratories to report sensitive and accurate results to doctors and also a decrease in turnaround times, low contamination risk, ease of performance, speed, manipulation of smaller volumes of liquid as well as the facility to have lower detection limits and reduce cost per test. The main aim for performing microbiology nucleic acid tests (NAT) is to provide time bound and accurate result for expert patient care at a reasonable cost. Rapid results obtained by NAT contribute to improvements in patient care. Polymerase chain reaction (PCR), real-time PCR (rt PCR) or nucleic acid sequence-based amplification $(\mathrm{NASBA})^{2}$ for detection, genotyping and quantification of virus have high sensitivity and reproducibility, and a broad dynamic range ${ }^{3}$. Identification of active infection by detection of viral messenger RNA (mRNA) through NASBA assays is done but quantitative real-time PCR technique is the most widely used tests in clinical virus diagnosis ${ }^{4}$. The main aim of this review is to focus on the appliance of molecular technology in the clinical virology laboratory.

\section{NON AMPLIFIED NUCLEIC ACID PROBES}

Nucleic acid probes or hybridization probe is a fragment of DNA or RNA of variable length (usually $100-1000$ bases long) virology laboratories.

Key Words: PCR, Probes, NASBA assays, NAT, Diagnosis. ${ }^{*}$ Correspondence to:

Dr. B. K. Bajaj, PhD,

Senior Assistant Professor,

School of Biotechnology, University of Jammu, Jammu, India.

Article History:

Received: 23-08-2016, Revised: 08-09-2016, Accepted: 14-09-2016

\begin{tabular}{|l|c|}
\hline \multicolumn{2}{|c|}{ Access this article online } \\
\hline $\begin{array}{l}\text { Website: } \\
\text { www.ijmrp.com }\end{array}$ & Quick Response code \\
\hline DOI: & \\
10.21276/ijmrp.2016.2.5.001 & \\
\hline
\end{tabular}

labeled radioactively, enzymatically or with chemiluminiscent molecules that bind complementary nucleic acid sequence of viruses $^{5}$. Despite having been around for many years, hybridization assays are still not in common use in the clinical virology laboratory. The main reason is that its sensitivity is not usually higher than far simpler conventional virological techniques such as cell culture and viral antigen detection. In general, these techniques have a poor analytical sensitivity, so they could be used only to those situations in which the number of microorganisms is large.

\section{AMPLIFIED NUCLEIC ACID TECHNIQUES}

The development of PCR led to the beginning of molecular diagnostics. This is the most widely used nucleic acid amplification technique, although several other methodologies have also been developed. These techniques are target, signal and probe amplification based.

\section{SIGNAL AMPLIFICATION TECHNIQUES}

These techniques do not replicate the nucleic acid detected in the assay but rather use amplification of a signal generated by detection of the target sequence. In signal amplification assays, the signal is directly proportional to the amount of the target sequence present in the clinical specimen, reducing false-positive results due to cross contamination; also, the development of quantitative assays is more reliable. 


\section{bDNA ASSAYS}

The branched or bDNA signal amplification system consists of a series of hybridization steps resulting in a "sandwich" complex of probes and target sequence with a branched structure Branched DNA (bDNA) technology has been integrated into commercial assays by Chiron Corporation uses multiple DNA probes, for detection, and then for increasing potential binding sites from which the reporter signal is eventually generated. The approach although less sensitive than target amplification has several advantages as the assay format is compatible to routine high throughput testing and not prone to false positives caused by cross contamination 6 . Importantly, bDNA assays are capable of detecting a broader range of viral genotypes than PCR based assays, which with their more limited number of target sequences have a restricted specificity: potentially a problem when detecting viral RNA genomes, which can show high sequence heterogeneity.

\section{HYBRID CAPTURE ASSAYS}

The Digene HPV Test using Hybrid Capture is a nucleic acid hybridization microplate assay with signal amplification through chemiluminescence. The target DNA is hybridized with a specific HPV RNA probe cocktail and the resultant RNA:DNA hybrids are coated with antibodies specific for RNA:DNA hybrids. Immobilized hybrids are then reacted with alkaline phosphatase-conjugated antibodies specific for the RNA:DNA hybrids and detected with a chemiluminescent substrate. Several alkaline phosphatase molecules are conjugated to each antibody. Multiple conjugated antibodies bind to each captured hybrid, resulting in substantial signal amplification. As the substrate is cleaved by the bound alkaline phosphatase, light is emitted that is measured as relative light units (RLUs) on a luminometer?

\section{TARGET AMPLIFICATION TECHNIQUES}

These techniques are enzyme-mediated processes, in which the enzymes synthesize several copies of target nucleic acid. The detection of amplified products is by two oligonucleotide primers that bind to complementary sequences. The final result is the production of millions of copies of the targeted sequence. There is possibility of contamination, so false positive results must be reduced through special laboratory design, practices and workflow.

\section{PCR TECHNIQUES}

Polymerase chain reaction (PCR) is a patented procedure developed by Kary Mullis in 1983, by which minute quantities of DNA or RNA can be enzymatically amplified using DNA polymerase. All the steps of PCR are performed on the reaction mixture consisting of target DNA, primer pairs, thermostable DNA polymerase, deoxynucleotides (dATP, dTTP, dGTP \& dCTP), buffer and $\mathrm{Mg}$ salt in the same test tube. The reaction mixture is heated and cooled during several cycles in a programmable thermal cycler, and after $n$ cycles the target sequence can be amplified $2 n$-fold. After the PCR reaction, the detection of product of amplification should be done by means several techniques ${ }^{8}$.

\section{REVERSE TRANSCRIPTASE-PCR}

Detection of viral nucleic acid (of RNA viruses) in clinical specimen and to prepare cDNA library of mRNA, a complimentary copy of RNA is made using enzyme reverse transcriptase (RT). Using a gene specific or oligo-dT primer mRNA RT synthesizes cDNA strand by adding complementary base pairs. Certain RT enzymes do not function beyond the non-stringent hybridization temperature of $42^{\circ} \mathrm{C}$ moreover single stranded RNA sometimes forms stable secondary structures and hamper conversion of RNA into $\mathrm{CDNA}^{9}$. A thermostable RT along with raised temperature not only increases the stringency of hybridization but also disrupts any secondary structures that might have formed. A recombinant DNA polymerase derived from Thermus thermophilus (Tth pol) has both polymerase as well as RT activity in the presence of $\mathrm{Mn}^{2+}$. Commercially kits are available for detection of HCV RNA and for quantitation of HIV-1 and HCV RNA in clinical specimens (Roche Diagnostics, Indianapolis, IND).

\section{NESTED PCR}

Nested polymerase chain reaction (Nested PCR) is a modification of polymerase chain reaction intended to reduce non-specific binding in products due to the amplification of unexpected primer binding sites. It uses two sets of amplification primers the first set amplifies a target sequence and the second set amplifies a region within the first target sequence. Essentially, this involves amplification of a sequence internal to an amplicon. Because the production of second amplicon depends on the successful production of the first amplicon, production of second amplicon automatically validates the accuracy of the first amplicon. Nested PCR may be performed in a single tube method or two-tube method. In the single tube method, both the external and internal primer sets are added at the same time and accomplish either by physical separation of two sets of reaction or by difference in annealing temperature of primers.

In the two tube procedure, target is amplified using only the outer primer sets after 25-30 PCR cycles. After this, the tube is opened and the mixture is transferred to another tube containing inner primer sets which hybridizes to the amplicon generated using outer primes. After running 25-30 cycles, the products are analyzed by standard methods ${ }^{10}$.

Nested PCR makes the reaction very specific and alleviates false positive reactions that may occur with other PCR systems. The high rate of contamination is a matter of concern

\section{MULTIPLEX PCR}

Multiplex PCR, is a widely used technique for simultaneous amplification of two or more unique target sequences. It can be used in diagnostic assays that use one set of primers to amplify an internal control to verify the integrity of the PCR while the second set of primer is targeted to DNA sequence of interest. Absence of control amplicon indicates that PCR conditions were not met and the PCR may have to be repeated. Multiplex PCR can also be used to test for different organism on a single specimen ${ }^{11}$. As an extension to the practical use of PCR, this technique has the potential to produce considerable savings in time and effort within the laboratory without compromising on the utility of the experiment

\section{REAL TIME PCR}

A real-time polymerase chain reaction is a laboratory technique of molecular biology based on the polymerase chain reaction (PCR) which monitors the amplification of a 
targeted DNA molecule during the PCR, i.e. in real-time, and not at its end, as in conventional PCR. Real-time PCR can be used quantitatively (Quantitative real-time PCR), semi-quantitatively, i.e. above/below a certain amount of DNA molecules (Semi quantitative real-time PCR).

An adapted thermal cycler is used to irradiate the samples with ultraviolet light, and the resulting fluorescence is detected with a computer-controlled cooled CCD camera. Amplification produces increasing amounts of double-stranded DNA, which binds ethidium bromide, resulting in an increase in fluorescence. By plotting the increase in fluorescence versus cycle number, the system produces amplification plots that provide quantitative picture of the PCR process.

The principal drawback of intercalator-based detection of PCR product accumulation is that both specific an nonspecific products generate signal. Subsequent improvements in Real-time systems for PCR were probe-based, rather than intercalator-based PCR product detection ${ }^{12}$.

Real-time RT-PCR has great advantages for estimating transcript levels in a variety of situations. The advantages include relative rapid assay times, reliability and ease of performing analyses. In contrast, competitive PCR is a very labour intensive procedure requiring a few days to generate useful data.

The advantage of fluorogenic probes over DNA binding dyes is that specific hybridization between probe and target is required to generate fluorescent signal. Thus, with fluorogenic probes, nonspecific amplification due to mis-priming or primer-dimer artifact does not generate signal. Another advantage of fluorogenic probes is that they can be labeled with different, distinguishable reporter dyes. By using probes labeled with different reporters, amplification of two distinct sequences can be detected in a single PCR reaction. The disadvantage of fluorogenic probes is that different probes must be synthesized to detect different sequences ${ }^{13}$.

\section{STRAND DISPLACEMENT AMPLIFICATION (SDA)}

SDA is an isothermal, in vitro nucleic acid amplification technique and relies on a the ability of Hincll to nick the unmodified strand of a hemiphosphorothioate form of its recognition site, and the ability of exonuclease deficient klenow (exo- klenow) to extend the 3'end at the nick and displace the downstream DNA strand. Exponential amplification results from coupling sense and antisense reactions in which strands displaced from a sense reaction serve as target for an antisense reaction and vice versa ${ }^{14}$. The most active current use of strand displacement amplification (SDA) is in identifying infectious agents such as tuberculosis for the purpose of diagnosing disease. Many other infections can be rapidly identified with these methods Tuberculosis, Chlamydia, Mycoplasma, H. influenzae, Gonorrhea, and Legionella, Shigella, and Salmonella species. Research is also focused on viruses, such as HIV, herpes, hepatitis, as well as candidal fungal infections.

\section{PROBE AMPLIFICATION TECHNIQUES}

In probe amplification procedures, the number of target nucleic acid sequences in a sample is not changed. Synthetic probes that are specific to the target sequences bind to the target where the probes themselves are amplified. These methods differ from those that use target amplification in which the amplification products contain only a sequence present in the initial probes. Ligase chain reaction (LCR), strand displacement amplification (SDA), and $\mathrm{Q} \beta$ replicase are the three major procedures that involve the amplification of probe sequences.

\section{CLEAVASE-INVADER TECHNOLOGY}

Clevase Invader technology relies on probe amplification method with specific recognition of a particular DNA structure by Cleavage enzymes, a class of naturally occurring and engineered nucleases that cleave unpaired regions (also called flaps) of DNA, which form when the 59 end of a DNA sequence overlaps the hybridization site of the 39 end of an upstream oligonucleotide by at least $1 \mathrm{bp}$.

Detection is accomplished through a fluorescence resonance energy transfer (FRET) mechanism. During the assay, when cleavage separates the donor fluorophore from the acceptor dye, quenching is minimized and a fluorescent signal is generated. The first-generation Invader assay has been reported and secondgeneration Invader assays developed which offer better precision and accuracy.

Assays based on Invader technology provide an uncomplicated, homogeneous platform sensitive enough to allow direct detection from genomic DNA without the need for PCR amplification and specific enough to discriminate single-base pair changes ${ }^{15}$. This single technology can be used to diagnose a wide spectrum of genetically based diseases, to detect specific single nuclear polymorphisms, to quantitate viral loads, and to test for drug resistance/mutations, providing a flexible platform with a wide breadth of applications for the clinical setting.

\section{LIGASE CHAIN REACTION}

The principle of LCR is based in part on the ligation of two adjacent synthetic oligonucleotide primers, which uniquely hybridize to one strand of the target DNA. This single base-pair difference may define two different alleles, species, or other polymorphisms correlated to a given phenotype. If the target nucleotide at that site complements the nucleotide at the $3^{\prime}$ end of the upstream primer, the two adjoining primers can be covalently joined by the ligase.

The unique feature of LCR is a second pair of primers, almost entirely complementary to the first pair, that are designed with the nucleotide at the $3^{\prime}$ end of the upstream primer denoting the sequence difference ${ }^{16}$. In a cycling reaction, using a thermostable DNA ligase, both ligated products can then serve as templates for the next reaction cycle, leading to an exponential amplification process analogous to PCR amplification. LCR assays have been developed for the detection of genetic diseases as well as for the detection of bacteria and viruses.

\section{CYCLING PROBE TECHNOLOGY}

Cycling probe technology (CPT) is a simple signal amplification method that operates under isothermal conditions for the detection of specific target DNA sequences. CPT uses a chimeric DNARNA-DNA probe that is cut by RNase $\mathrm{H}$ when bound to its complementary target sequence ${ }^{17}$. In some applications, CPT offers an alternative to PCR. However, unlike PCR, CPT does not generate multiple copies of the target DNA itself, and the amplification of the signal is linear, in contrast to the geometric amplification of the target DNA in PCR. 


\section{NEW MOLECULAR TESTS FOR VIROLOGICAL DIAGNOSIS MICROARRAYS}

An array is an orderly arrangement of samples where matching of known and unknown DNA samples is done based on base pairing rules. An array experiment makes use of common assay systems such as microplates or standard blotting membranes. The sample spot sizes are typically less than 200 microns in diameter and usually contain thousands of spots called probes.

These probes are of known identity and can be be DNA, CDNA, or oligonucleotides. These are used to determine complementary binding of the unknown sequences thus allowing parallel analysis for gene expression and gene discovery. An experiment with a single DNA chip can provide information on thousands of genes simultaneously. An orderly arrangement of the probes on the support is important as the location of each spot on the array is used for gene identification. The diagnosis of viruses is tedious process due to the inherent sequence diversity and ongoing emergence of novel viral pathogens, such as SARS coronavirus and 2009 pandemic H1N1 influenza virus. A pan-viral microarray platform called the Virochip with the capacity to detect all known viruses as well as novel variants on the basis of conserved sequence homology ${ }^{18}$ has been developed. A full spectrum of viruses associated with respiratory infections, including cases of unexplained critical illness in hospitalized patients, with a sensitivity equivalent to or superior to conventional clinical testing novel viruses, including the SARS coronavirus, a novel rhinovirus clade5, XMRV (a retrovirus linked to prostate cancer), avian bornavirus (the cause of a wasting disease in parrots), and a novel cardiovirus in children with respiratory and diarrheal illness ${ }^{19}$.

\section{MULTIPLEXED MICROSPHERE-BASED ARRAY}

Over the past decade, soluble microsphere (bead)-based arrays have become an increasingly popular alternative for quantifying Abs. and are an advantageous replacement of ELISA. As they can be easily multiplexed, thereby reducing the sample volume required to evaluate multiple Abs, provide soluble-phase binding of antigen-antibody pairs, and offer a vastly increased dynamic range compared to the ELISA optical density scale. One of the most popular microsphere-based assays relies on Luminex XMAP technology (Luminex Corp., Austin, TX). In this system, various microsphere populations are internally labeled with varying concentrations of fluorescent dyes, giving each microsphere type in the array a unique fluorescence signature that can be identified with an appropriate instrument, such as the Bio-Plex 200 System plate reader (BioRad, Hercules, CA). As a result, when proteins of interest are covalently linked to unique bead populations, up to 100 different analytes can theoretically be measured in a single Bio-Plex plate well. Such multiplexed assays are particularly useful when simultaneous measurement of numerous analytes in a given sample is desired, as is often the case with cytokines, for which numerous assays have been developed ${ }^{20}$. Multiplexed bead-based immunoassays have been established and commercial kits are available for simultaneous measurement of specific Abs against various disease, atopic, autoimmune, and cancer related antigens. These assays have been employed to measure analytes in cultured cells or in various bodily fluids such as serum, semen, and cervical mucus from humans or numerous experimental animals.

\section{ISOTHERMAL AMPLIFICATION}

PCR is a recognized approach to amplify a specific DNA sequence involving reiterative cycling of a reaction cocktail between different temperatures to achieve amplification. Besides PCR, there are other methods of sequence-specific DNA amplification which often do not require changing the reaction temperature and are, therefore, often referred to as sequencespecific isothermal amplification protocols. Isothermal amplification protocols are varied and have varied advantages. However, some common advantages are that isothermal techniques are extremely fast and they do not require thermocyclers. The main isothermal methods include loopmediated isothermal amplification (LAMP) and helicasedependent amplification (HDA) ${ }^{21}$.

\section{LOOP-MEDIATED ISOTHERMAL AMPLIFICATION (LAMP)}

LAMP is a simple, rapid, cost effective and specific amplification method developed by Eiken Chemical Co., Ltd. characterized by the use of 4 different primers designed specifically to recognize 6 different regions on the target gene while the reaction continues at a specific temperature by strand displacement reaction. Amplification and detection of gene can be completed in a single step, by incubating the mixture of samples, primers, DNA polymerase with strand displacement activity and substrates at a constant temperature (about $65^{\circ} \mathrm{C}$ ). It provides high amplification efficiency, with DNA being amplified $10^{9}-10^{10}$ times in 15-60 minutes. Because of its high specificity, the presence of amplified product can indicate the presence of target gene ${ }^{22}$.

LAMP is a relatively new DNA amplification technique, which due to its simplicity, ruggedness, and low cost could provide major advantages. LAMP holds the potential to be used as a simple screening assay in the field or at the point of care by clinicians. Because LAMP is isothermal, which eradicates the need for expensive thermocyclers used in conventional PCR, it may be a particularly useful method for infectious disease diagnosis in low and middle income countries. While, LAMP is widely being studied for detecting infectious diseases such as tuberculosis, malaria and sleeping sickness ${ }^{23}$. In developing regions, it has yet to be extensively validated for other common pathogens.

\section{HELICASE-DEPENDENT AMPLIFICATION (HDA)}

HAD is a novel method for the isothermal in vitro amplification of nucleic acids. The HDA reaction is similar to conventional PCR but utilizes the action of a thermostable helicase enzyme which separates the deoxyribonucleic acid (DNA) strands and also selectively amplifies a target sequence by extension of two oligonucleotide primers. This allows DNA amplification without the need for thermal cycling. The helicase used in HDA is a helicase super family II protein obtained from a thermophilic organism, Thermoanaerobacter tengcongensis (TteUvrD). This thermostable helicase is capable of unwinding blunt-end nucleic acid substrates at elevated temperatures $\left(60^{\circ}\right.$ to $\left.65^{\circ} \mathrm{C}\right)$. The HDA reaction can also be coupled with reverse transcription for ribonucleic acid (RNA) amplification ${ }^{24}$. The products of this reaction can be detected during the reaction using fluorescent probes when incubations are conducted in a fluorimeter. Alternatively, products can be detected after amplification using a disposable amplicon containment device that contains an embedded lateral flow strip. 


\section{CONCLUSION}

Clinical virology laboratory assists clinicians in the diagnosis and treatment of viral diseases, and supports infection control specialists in their tasks. There is always a need for a rapid identification of the etiological microorganisms for an effective patient management.

Since its development molecular techniques has revolutionized viral diagnosis in clinical laboratories. In the majority of them, these methods have been introduced for routine diagnosis, but some of those are being used only in reference settings Molecular techniques are the preferred diagnostic methods owing to their high sensitivity and specificity, rapidity and possibility of automation. Besides, this automation permits performance of assays and helps improve the patient diagnosis.

\section{ACKNOWLEDGEMENT}

We are thankful to School of Biotechnology, University of Jammu, Jammu.

\section{REFERENCES}

1. Hayden R, Persing DH. Diagnostic molecular microbiology review. Curr Clin Top Infect Dis 2001; 21: 323-48.

2. Van Belkum A, Niesters HG. Nucleic acid amplification and related techniques in microbiological diagnosis and epidemiology. Cell Mol Biol 1995; 41: 615-23.

3. Ebner K, Rauch M, Preuner S, Lion T. Typing of human adenoviruses in specimens from immunosuppressed patients by PCR-fragment length analysis and real-time quantitative PCR. J Clin Microbiol 2006; 44: 2808-15.

4. Atkinson C, Emery VC. Cytomegalovirus quantification: where to next in optimising patient management? J Clin Virol 2011; 51: 223-8.

5. Arnold LJ Jr., Hammond PW, Wiese WA, Nelson NC. Assay formats involving acridinium ester-labeled DNA probes. Clin Chem 1989; 35: 1588-94.

6. Nolte FS. Branched DNA signal amplification for direct quantitation of nucleic acid sequences in clinical specimens. Adv Clin Chem 1999; 33:201-35.

7. Poljak M, Marin IJ, Seme K, Vince A. Hybrid Capture II HPV Test detects at least 15 human papillomavirus genotypes not included in its current high-risk probe cocktail. J Clin Virol. 2002; 25 (3): S89-97.

8. Steven J Read, David Burnett, Colin G Fink. Molecular techniques for clinical diagnostic virology. J Clin Pathol 2000; 53:502-506.

9. Carlos Ferreira Dos Santos, Vivien Thiemy Sakai, Maria Aparecida De Andrade Moreira Machado, Daniela Nicole Schippers, Andrew Seth Greene. Reverse transcription and polymerase chain reaction: principles and applications in dentistry.

J Appl Oral Sci 2004; 12(1): 1-11

10. Rodney M. Ratcliff, Grace Chang, TuckWeng Kok, and Theo P. Sloots. Molecular Diagnosis of Medical Viruses. Curr. Issues Mol. Biol. 9: 87-102.

11. Chamberlain JS, Gibbs RA, Rainer JE, Nguyen PN, Caskey CT.Deletion screening of the Duchenne muscular dystrophy locus via multiplex DNA amplification. Nucleic Acids Res 1988; 16 : 11141-56.

12. Hiyoshi, M., and Hosoi, S. (1994). Assay of DNA denaturation by polymerase chain reaction-driven fluorescent label incorporation and fluorescence resonance energy transfer. Anal. Biochem. 221, 306- 311.

13. Manit Arya, Iqbal S Shergill, M Williamson, L Gommersall, N Arya and Hitendra $\mathrm{RH}$ Patel. Basic principles of real-time quantitative PCR. Expert Rev. Mol. Diagn 2005. 5(2).

14. Md Fakruddin, Khanjada Shahnewaj Bin Mannan, Abhijit Chowdhury, Reaz Mohammad Mazumdar, Md. Nur Hossain,Sumaiya Islam, and Md. Alimuddin Chowdhury. Nucleic acid amplification: Alternative methods of polymerase chain reaction. J Pharm Bioallied Sci. 2013 Oct-Dec; 5(4): 245-252.

15. Michael Olivier. The Invader ${ }^{\circledR}$ assay for SNP genotyping. Mutat Res. 2005 June 3; 573(1-2): 103-110.

16. Martin Wiedmann, Wendy J Wilson, John Czajka, Jianying Lou, Francis Barany and Carl A. Batt. Ligase Chain Reaction (LCR): Overview and Applications. PCR Methods and Applications.3:S51-S64, 1994 by Cold Spring Harbour Laboratory. 17. S. Warnon, N. Zammatteo, I. Alexandre, C. Hans and J. Remacle. Colorimetric Detection of the Tuberculosis Complex Using Cycling Probe Technology and Hybridization in Microplates. BioTechniques; June 2000; 28:1152-1160.

18. Wang D. Microarray-based detection and genotyping of viral pathogens. Proc Natl Acad Sci U S A. 2002; 99:1 5687-15692.

19. Chiu CY. Utility of DNA microarrays for detection of viruses in acute respiratory tract infections in children. $\mathrm{J}$ Pediatr. 2008;153:76-83.

20. Rebecca L.R. Powell, lan O, Ross W. L, Christopher L. Parks, C. Richter King, Adrian B. McDermott, and Gavin Morrow. A Multiplex Microsphere-Based Immunoassay Increases the Sensitivity of SIV-Specific Antibody Detection in Serum Samples and Mucosal Specimens Collected from Rhesus Macaques Infected with SIVmac239. BioResearch Open Access 2013; 2(3).

21. Sidoti F, Bergallo M, Costa C, Cavallo R. Alternative molecular tests for virological diagnosis. Mol Biotechnol. 2013; 53(3):352-62. 22. Notomi T, Okayama H, Masubuchi $H$, Yonekawa T, Watanabe $\mathrm{K}$, Amino N, Hase T. Loop mediated isothermal Amplification of DNA. Nucleic Acids Res. 2000 Jun 15;28(12):E63.

23. Macarthur G. Global health diagnostics: research, development and regulation. Academy of Medical Sciences Workshop Report. Academy of Medical Sciences (GreatBritain). 2009: ISBN 978-1-903401-20-0. http://www.acmedsci.ac.uk/viewFile/publicationDownloads/127607 104184.pdf

24. Myriam V, Yan $\mathrm{Xu}$ and Huimin K. Helicase-dependent isothermal DNA amplification EMBO reports 2004: 5 (8):795.

\section{Source of Support: Nil. Conflict of Interest: None Declared.}

Copyright: (c) the author(s) and publisher. IJMRP is an official publication of Ibn Sina Academy of Medieval Medicine \& Sciences, registered in 2001 under Indian Trusts Act, 1882.

This is an open access article distributed under the terms of the Creative Commons Attribution Non-commercial License, which permits unrestricted non-commercial use, distribution, and reproduction in any medium, provided the original work is properly cited.

Cite this article as: Konika Razdan, Varshiesh Raina and Bijendra K Bajaj. Diagnosis of Medically Important Viruses: A Molecular Approach. Int J Med Res Prof. 2016; 2(5):1-5. 